Département de sciences économiques

2004-12

\title{
The Progressivity of Equalization Payments in Federations
}

GRAVEL, Nicolas

POITEVIN, Michel 


\section{Département de sciences économiques}

Université de Montréal

Faculté des arts et des sciences

C.P. 6128, succursale Centre-Ville

Montréal (Québec) H3C 3J7

Canada

http://www.sceco.umontreal.ca

SCECO-information@UMontreal.CA

Téléphone : (514) 343-6539

Télécopieur : (514) 343-7221

Ce cahier a également été publié par le Centre interuniversitaire de recherche en économie quantitative (CIREQ) sous le numéro 14-2004.

This working paper was also published by the Center for Interuniversity Research in Quantitative Economics (CIREQ), under number 14-2004.

ISSN 0709-9231 


\title{
The Progressivity of Equalization Payments in Federations*
}

\author{
Nicolas Gravel ${ }^{\dagger}$ and Michel Poitevin ${ }^{\ddagger}$
}

August 25, 2004

\begin{abstract}
We investigate the conditions under which an inequality averse and additively separable welfarist constitution maker would always choose to set up a progressive equalization payments scheme in a federation with local public goods. A progressive equalization payments scheme is defined as a list of per capita net (possibly negative) subsidies - one such net subsidy for every jurisdiction - that are decreasing with respect to jurisdictions per capita wealth. We examine these questions in a setting in which the case for progressivity is a priori the strongest, namely, all citizens have the same utility function for the private and the public goods, inhabitants of a given jurisdiction are all identical, and they are not able to move across jurisdictions. We show that the constitution maker favors a progressive equalization payments scheme for all distributions of wealth and all population sizes if and only if its objective function is additively separable between each jurisdiction's per capita wealth and number of inhabitants. When interpreted as a mean of order $r$ social welfare function, this condition is shown to be equivalent to additive separability of the individual's indirect utility function with respect to wealth and the price of the public good. Some implications of this restriction to the case where the individual's direct utility function is additively separable are also derived.
\end{abstract}

${ }^{*}$ We are indebted to Chuck Blackorby, Robin Boadway and Philippe Michel for helpful discussions. We acknowledge financial support from CRSH, the CIREQ and the Département de sciences économiques, Université de Montréal.

JEL classification numbers: D630, H100, H200,H210, H410, H700, H730, H770.

Key words: Local public goods, federations, redistribution, progressivity.

†IDEP-GREQAM and Université de la Méditerranée, Centre de la Vieille Charité, 2, rue de la Charité, 13002 Marseille Cedex, gravel@ehess.univ-mrs.fr.

${ }^{\ddagger}$ CIREQ, CIRANO and Département de sciences économiques, Université de Montréal, C.P. 6128, succursale Centre-ville, Montréal, QC, Canada H3c 3J7; Tel.: (514) 343.6543; Fax: (514) 343.7221; michel.poitevin@umontreal.ca. 


\section{Introduction}

Many federal countries have developed equalization payments schemes by which a central government transfers money between jurisdictions. For instance these equalization payments are entrenched in the Canadian constitution. They also underlie the design of the European Funds for Structural Development which are given to specific regions suffering from economic backwardness. The alleged purpose of these schemes is, as their name suggests, to equalize citizens' access to public services across jurisdictions. It is usually thought that these transfers should somehow correct for the unequal distribution of wealth across jurisdictions. More specifically, most equalization payments schemes that we are aware of are explicitly progressive: they are designed in such a way that the (net) per capita subsidy received by a jurisdiction is decreasing with respect to its per capita wealth.

There are, of course, many reasons to question the soundness of this progressivity from a normative viewpoint. One such reason is cross-jurisdiction taste differences. Why should the inhabitants of a jurisdiction who like the public good and who decide to contribute extensively to its financing be required to transfer money to people living in a slightly poorer

jurisdiction who care very little about the public good and have all their money available for private consumption?

Another obvious source of skepticism with respect to progressive equalization systems is within-jurisdiction heterogeneity. Suppose jurisdiction $A$ has a slightly higher per capita wealth than jurisdiction $B$ but that the distribution of wealth within $A$ is much more unequal than $B$. Suppose in particular that a significant fraction of $A$ 's population is extremely poor while nobody experiments severe poverty in $B$. There is then no reason to expect a transfer of money from $A$ to $B$. As a matter of fact, standard inequality aversion considerations, such as those underlying the ranking of Lorenz curves, could very well recommend a transfer from $B$ to $A$ in a case like this.

A third easy case that can be made against progressivity arises if mobility across jurisdictions is high. If citizens can easily move from one jurisdiction to the next, then progressive 
equalization payments may not be sustainable because it may induce citizens from donator jurisdictions who make transfer payments (and who therefore receive less public good than the tax they pay) to move to recipient ones. In order to prevent such migration, the constitution maker may have to limit the progressivity of its scheme. ${ }^{1}$

But suppose we abstract from these three reasons that mitigate the appeal of progressivity of equalization payments schemes in federations. Wouldn't progressivity become defensible then? The aim of this note is to provide a negative answer to this question. More specifically, we consider an arbitrary federation populated by a given number of individuals who have the same utility function for one private good and one local public good. These individuals are partitioned into a given number of jurisdictions according to their wealth. All individuals within a jurisdiction have the same wealth, and individuals are not allowed to move across jurisdictions. In this stylized world, we examine the type of equalization payments systems that a "constitution maker" would adopt if its objective was the maximization of a symmetric, quasi-concave and additively separable function of the citizens' well-being. ${ }^{2}$

We show that, even in this a priori favorable case, the conditions for the optimal equalization payments scheme to be progressive for all distributions of wealth and individuals between jurisdictions are stringent. More specifically, we show that a necessary and sufficient condition that the objective function of the constitution maker must satisfy in order to always choose a progressive equalization payments scheme is to be additively separable with respect to a jurisdiction's per capita wealth and its number of inhabitants, a variable which corresponds to the (inverse of) the Lindahl price of the public good.

\footnotetext{
${ }^{1}$ It is not at all clear that allowing for cross-jurisdiction mobility would reduce the progressivity of the equalization payment scheme that a welfarist constitution maker would favor. If citizens living in relatively poor jurisdictions envy the package of public good and taxes of their wealthier neighbors, the constitution maker may have to increase progressivity in order to prevent the citizens of poorer jurisdictions to move to richer ones. The (difficult) analysis of the optimal equalization payments scheme when mobility is allowed across jurisdictions is the object of another paper (see Gravel and Poitevin (2004)).

${ }^{2}$ See Aczél and Pfingsten (1993) or Buhl and Pfingsten (1990) for an alternative normative approach to equalization payments in federations.
} 
The stringency of this condition can be appreciated better if the social welfare function used by the constitution maker to aggregate individuals' utilities is specialized somewhat. A specialization which seems natural in the redistributive context considered here is the mean of order $r$ family of social welfare functions which contains many well-known and widely used social welfare functions (such as utilitarianism, Rawls, Nash-Bernoulli, etc.) as special cases. If such a specialization is adopted, we show that the additive separability of the constitution maker's objective function implies the additive separability of the individual's indirect utility function between wealth and the (Lindahl) price of the public good. This is a condition that significantly restricts the kind of preferences that citizens are allowed to have over the public and private goods. For instance, if the direct utility function for the private and public goods is assumed to be additively separable itself, the additive separability of the citizen's indirect utility between wealth and the price of public good implies that the direct utility is logarithmic with respect to the public good.

That these restrictions be necessary and sufficient for a progressive equalization payments system to be deemed optimal from a welfarist point of view even in this stylized world are clear indications that progressivity is not a natural feature of an optimal equalization payments scheme. More fundamentally these results show that, when dealing with redistribution in multi-jurisdictions systems with public good provision, individual wealth is not the only variable of interest. Another one is the number of people living in the jurisdiction which contributes to reducing the jurisdiction's per capita cost of providing the public good. Clearly, jurisdictions with many inhabitants are able to afford a given amount of public good at a lower per capita tax cost. This advantage of large population jurisdictions over small population ones must be accounted for by the constitution maker when performing crossjurisdictions redistribution. Of course the specific nature of this account depends crucially upon the way by which the marginal social value of wealth, which the constitution maker seeks to equalize across jurisdictions, varies with the tax price of the public good. If the marginal social value of wealth increases with the tax price of the public good, then the constitution maker may be willing to transfer wealth from highly populated and relatively 
poor jurisdictions to richer but sparsely populated ones. Conversely, if the marginal social value of wealth decreases with respect to the tax price, the constitution maker may find appropriate to transfer wealth from lowly populated and poor jurisdictions to richer and heavily populated ones. In each of these cases, the constitution maker may find appropriate to depart from progressivity. It is only when the marginal social value of wealth is independent from the tax price - which arises if the social objective function is additively separable between the tax price and wealth - that the constitution maker always finds progressivity to be optimal.

The next section introduces the model and proves the main result. Section 3 interprets the result in the specific case where the constitution maker's objective is a mean of order $r$ of the citizens' utility, and examines the implications of the result for the case where households' direct utility is assumed to be additively separable. Section 4 concludes.

\section{The model and main result}

\subsection{The model}

We consider a country populated by $n \in \mathbb{N}_{++}$households living in $k \in\{1, \ldots, n\}$ a priori given jurisdictions. There are $n_{j}$ households who live in jurisdiction $j(j=1, \ldots, k)$ so that

$\sum_{j=1}^{k} n_{j}=n$. A household living in jurisdiction $j$ has a private wealth $\omega_{j}$ which it uses to pay taxes and to make private consumption. Jurisdictions are labeled in such a way that $\omega_{1} \geq \omega_{2} \geq \ldots \geq \omega_{k}$. Households derive utility from a single local public good (whose quantity is denoted by $z$ ) and from private consumption (whose quantity is denoted by c). Specifically, all households in the country convert alternative bundles of public and private good into well-being by the same strictly concave, monotonically increasing and twice continuously differentiable utility function $U: \mathbb{R}_{+}^{2} \rightarrow \mathbb{R}$. We shall also sometimes assume, notably in section 3 , that, in addition to the above properties, $U$ is additively separable so that it can 
be written, for every bundle $(\bar{z}, \bar{c}) \in \mathbb{R}_{+}^{2}$, as

$$
U(\bar{z}, \bar{c})=f(\bar{z})+h(\bar{c})
$$

for some twice continuously differentiable functions fand $h$ from $\mathbb{R}_{+}$to $\mathbb{R}$. We denote by $V$ the indirect utility function defined, for every $\left(p_{z}, p_{c}, R\right) \in \mathbb{R}_{++}^{3},{ }^{3}$ by

$$
V\left(p_{z}, p_{c}, R\right)=\max _{z, c} U(z, c) \text { subject to } p_{z} z+p_{c} c \leq R
$$

We also denote by $z^{M}\left(p_{z}, p_{c}, R\right)$ and $c^{M}\left(p_{z}, p_{c}, R\right)$ the (Marshallian) demands for the public good and private consumption (respectively) when the prices for these two goods are $p_{z}$ and $p_{c}$ and when the wealth of the household is $R$. These Marshallian demands are defined as usual as the solution of program (1). Given the assumptions imposed on $U$, we know that the Marshallian demands and the indirect utility function are differentiable functions of prices and wealth. We denote by $\mathcal{U}$ the class of all direct utility functions that satisfy all these properties and by $\mathcal{U}_{A}$, the subset of $\mathcal{U}$ consisting of those functions that are additively separable.

The local public good is purchased at a price $p>0$ and its purchase is financed by taxation. Tax rates are allowed to differ across jurisdictions. We consider the case where households are not allowed to move across jurisdictions. ${ }^{4}$ Moreover, we allow the federal government to redistribute purchasing power across jurisdictions in order to harmonize private good and public good consumption. In this setting, if $T_{j}=\omega_{j}-c_{j}$ denote the tax paid by a household living in jurisdiction $j$, the country's feasibility constraint writes:

$$
\sum_{j=1}^{k} p z_{j} \leq \sum_{j=1}^{k} n_{j} T_{j} .
$$

\footnotetext{
${ }^{3}$ We restrict the domain of admissible prices and wealth to $\mathbb{R}_{++}^{3}$.

${ }^{4}$ The more realistic situation where households are allowed to move across jurisdictions is examined in a companion paper (see Gravel and Poitevin (2004)).
} 


\subsection{Conditions for a progressive equalization payments scheme}

Assume that the constitution maker is welfarist in the sense that it ranks alternative allocations of tax and public good according to the value taken by a monotonically increasing function of the citizens' well-being. Welfarism is obviously not the only viewpoint for appraising normatively equalization payments in a federation (see, for instance, Aczél and Pfingsten (1993) or Buhl and Pfingsten (1990) for alternatives). Yet, it is a widely used ethics in economics and can be given convincing justifications (for example, see Blackorby, Bossert and Donaldson (2001)). This welfarist assumption amounts to say that the constitution maker chooses taxes and public good levels that solve the following program.

$$
\begin{array}{rl}
\max _{z_{1}, T_{1}, \ldots, z_{k}, T_{k}} & W\left(U\left(z_{1}, \omega_{1}-T_{1}\right), \ldots, U\left(z_{k}, \omega_{k}-T_{k}\right)\right) \\
\text { s.t. } & T_{j} \leq \omega_{j}, z_{j} \geq 0 \text { for all } j, \text { and } \\
& \sum_{j=1}^{k} p z_{j} \leq \sum_{j=1}^{k} n_{j} T_{j},
\end{array}
$$

for some continuous and monotonically increasing Bergson-Samuelson social welfare function $W: \mathbb{R}^{n} \rightarrow \mathbb{R}$.

Consider a solution $\left\{z_{j}^{*}, T_{j}^{*}\right\}_{j=1}^{k}$ to this program (which exists by virtue of Weierstrass Theorem). It is immediate to see that this solution satisfies the budget constraint (2) at equality. Let $s_{j}^{*}=p z_{j}^{*} / n_{j}-T_{j}^{*}$ denote the (possibly negative) net per capita subsidy received by a household of jurisdiction $j$. We characterize under which conditions a welfarist constitution maker would always find optimal to select net per capita subsidies that are progressive in the precise sense of being decreasing with respect to the per capita jurisdiction's wealth. For further reference, we give a formal definition of this notion of progressivity as follows.

Definition 1. An equalization payments system $s_{1}, \ldots, s_{k}$ is progressive if and only if $s_{j} \leq$ $s_{j+1}$ for all $j=1, \ldots, k-1$.

This definition of progressivity deserves, perhaps, a few comments. The common definition of progressivity of a tax system is that the ratio of the net taxes paid by a household 
over its income is non-decreasing with respect to income. Using this definition in our setting would amount to require that the ratio $s_{j} / \omega_{j}$ of the per capita net subsidy over the jurisdiction's per capita wealth to be decreasing with respect to per capita wealth. The justification usually given to this common definition of progressivity lies in the fact, apparently first established by Jakobsson (1976) (see also Eichhorn, Funke and Richter (1984), Moyes (1994) and Thon (1987)) that it is equivalent to requiring the relative Lorenz curve associated to the post-tax income distribution to be everywhere above that associated to the before-tax income distribution. As is well-known, the relative Lorenz curve associated to an income distribution is the graph of the function that maps every household's rank in the ordering of incomes to the fraction of the aggregate wealth held by all households in (weakly) lower ranks. Albeit there exists good arguments for using the relative Lorenz curve when appraising the impact of alternative policies on income inequalities, the relative conception of equality which underlies this curve has been the object of some criticism, notably by Kolm (1976). These criticisms have motivated the definition of an absolute Lorenz curve, studied in particular by Moyes (1987). The absolute Lorenz curve is the graph of the function that maps every household's rank into the difference between the aggregate wealth and the total income held by households. As it turns out, it is the criterion of absolute Lorenz domination that would justify the notion of progressivity considered in this paper. More specifically, as established by Moyes (1988) and Moyes (1994), requiring a tax system to always lead to a post-tax income distribution whose absolute Lorenz curve lies everywhere above that of the pre-tax income distribution is equivalent to requiring households' tax payments to be non-decreasing with respect to households' wealth.

While we are doing the analysis with the absolute notion of progressivity, it is an easy matter (see fact 1 below) to rephrase it in terms of the relative notion.

We start the analysis by establishing a simple (but useful) lemma ${ }^{5}$ which says that the social planner's problem (3) can be thought of as being solved in two steps: a first step in which the constitution maker chooses the per capita net subsidies $\left(s_{1}^{*}, \ldots, s_{k}^{*}\right)$ that maximize

\footnotetext{
${ }^{5}$ The proofs of all lemmas and theorems have been gathered in the Appendix.
} 
the composition of the social welfare function with the households' indirect utilities, and a second step where each jurisdiction $j$ 's household solves a fictitious standard consumer's problem of allocating optimally its private wealth and the net per capita subsidy between private consumption (purchased at a price of 1) and public good spending (purchased at price $\left.p / n_{j}\right)$.

Lemma 1. Let $U$ be a utility function in $\mathcal{U}$. Then the vector of local public goods and taxes $\left\{z_{j}^{*}, T_{j}^{*}\right\}_{j=1}^{k}$ defined, for every $j=1, \ldots, k$, by $z_{j}^{*}=z_{j}^{M}\left(p / n_{j}, 1, \omega_{j}+s_{j}^{*}\left(p, n_{1}, \omega_{1}, \ldots, n_{k}, \omega_{k}\right)\right)$ and $T_{j}^{*}=\omega_{j}-c_{j}^{M}\left(p / n_{j}, 1, \omega_{i}+s_{j}^{*}\left(p, n_{1}, \omega_{1}, \ldots, n_{k}, \omega_{k}\right)\right.$ where

$$
\begin{gathered}
\left\{s_{j}^{*}(\cdot)\right\}_{j=1}^{k} \in \arg \max _{s_{1}, \ldots, s_{k}} W\left(V\left(p / n_{1}, 1, \omega_{1}+s_{1}\right), \ldots, V\left(p / n_{k}, 1, \omega_{k}+s_{k}\right)\right) \\
\text { s.t. } \quad \sum_{j=1}^{k} n_{j} s_{j} \leq 0 \text { and } s_{j} \geq-\omega_{j} \text { for all } j,
\end{gathered}
$$

define a solution to the original program (3).

This lemma highlights the fact that, in a federation made of different homogeneous jurisdictions, jurisdiction's per capita wealth is not the only criterion used by the constitution maker to design equalization payment schemes. The constitution maker must also take into consideration the number of households living in a jurisdiction. The larger this number is, the lower is the per capita cost (or price) of providing one unit of public good in a jurisdiction. When redistributing wealth across jurisdictions, the constitution maker must take into consideration these cross-jurisdiction differences in the price of public good.

We want to know the conditions that the constitution maker's objective need to satisfy in order for the solution of (4) to be progressive for all distributions $\left\{n_{j}, \omega_{j}\right\}_{j=1}^{k}$ of population sizes and individual wealths.

To make this question somewhat interesting, it is natural to impose additional restrictions on the Bergson-Samuelson function used by the constitution maker. For progressivity is clearly not to be expected a priori from an arbitrary social welfare function which does not exhibit some inequality aversion. We therefore require $W$ to be quasi-concave and symmetric. 
We also require $W$ to be additively separable with respect to utilities. This latter assumption, which can be justified on normative grounds (see for instance Blackorby et al. (2001)), is made for simplicity. These three assumptions amount to say that $W$ can be defined by

$$
W\left(u_{1}, \ldots, u_{n}\right)=\sum_{i=1}^{n} g\left(u_{i}\right)
$$

for some monotonically increasing and continuous function $g: \mathbb{R} \rightarrow \mathbb{R}$ which needs to be concave if $W$ is to be quasi-concave. A nice example of a Bergson-Samuelson social welfare function which fits in this setting is the mean-of-order $r$ function where, for any $r \in-\infty, 1]$, $W_{r}: \mathbb{R}_{+}^{n} \rightarrow \mathbb{R}$ is defined by ${ }^{6}$

$$
\begin{aligned}
& W_{r}\left(u_{1}, \ldots, u_{n}\right)=\left[\sum_{j=1}^{n} U_{j}^{r}\right]^{\frac{1}{r}} \text { if } r \neq 0 \text { and } \\
& W_{r}\left(u_{1}, \ldots, u_{n}\right)=\sum_{j=1}^{n} \ln U_{j} \text { otherwise, }
\end{aligned}
$$

where the functions $g^{r}$ referred to in (5) can be defined by

$$
\begin{aligned}
& \left.\left.g^{r}(u)=u^{r} \text { if } r \in\right] 0,1\right] \\
& g^{0}(u)=\ln u \text { and } \\
& g^{r}(u)=-u^{r} \text { if } r \in-\infty, 0[.
\end{aligned}
$$

As is well known (and can be easily seen), the case where $r=1$ is that of a utilitarian constitution maker, while the limiting case of $r=-\infty$ is that which would correspond to an infinitely inequality averse Rawlsian one. We shall return to this example in the next section.

\footnotetext{
${ }^{6}$ The definition of this social welfare function requires individual utilities to be measured in positive units.
} See Blackorby and Donaldson (1982) for justifications and properties of this social welfare function. 
For a social welfare function satisfying (5), program (4) becomes

$$
\begin{aligned}
\max _{s_{1}, \ldots, s_{k}} & \sum_{j=1}^{k} n_{j} g\left(V\left(p / n_{j}, 1, \omega_{j}+s_{j}\right)\right) \\
\text { s.t. } & \sum_{j=1}^{k} n_{j} s_{j} \leq 0, \text { and } s_{j} \geq-\omega_{j} \text { for all } j .
\end{aligned}
$$

Defining the function $\Phi: \mathbb{R}_{++}^{2} \rightarrow \mathbb{R}$ by

$$
\Phi\left(p_{z}, R\right)=g\left(V\left(p_{z}, 1, R\right)\right)
$$

we can more compactly write (4) as

$$
\begin{aligned}
\max _{s_{1}, \ldots, s_{k}} & \sum_{j=1}^{k} n_{j} \Phi\left(p / n_{j}, \omega_{j}+s_{j}\right) \\
\text { s.t. } & \sum_{j=1}^{k} n_{j} s_{j} \leq 0, \text { and } s_{j} \geq-\omega_{j} \text { for all } j .
\end{aligned}
$$

This program has a unique solution $s_{j}^{*}($ for $j=1, \ldots, k$ ) (due to the strict concavity of $\Phi$ with respect to its second argument). This solution is, thanks to Berge's Maximum Theorem, a continuous function $s_{j}^{*}\left(p, n_{1}, \ldots, n_{k}, \omega_{1}, \ldots, \omega_{k}\right)$ of the $2 k+1$ parameters that define program (7). It is differentiable in those parameters if the objective function is twice differentiable. It follows in particular that the first-order conditions of (7) characterize any interior solution to this program.

The first-order conditions are

$$
\begin{aligned}
\forall h, j \in\{1, \ldots, k\}: \Phi_{R}^{j *}(\cdot) & \equiv \Phi_{R}^{h *}(\cdot), \\
\sum_{j=1}^{k} n_{j} s_{j}^{*}\left(p, n_{1}, \ldots, n_{k}, \omega_{1}, \ldots, \omega_{k}\right) & \equiv 0
\end{aligned}
$$

where, for every jurisdiction $j$,

$$
\Phi_{R}^{j *}(\cdot)=\frac{\partial \Phi\left(p / n_{j}, \omega_{j}+s_{j}^{*}\left(p, n_{1}, \ldots, n_{k}, \omega_{1}, \ldots, \omega_{k}\right)\right)}{\partial R} .
$$


Differentiating these conditions with respect to $\omega_{h}$, one obtains:

$$
\frac{\partial s_{j}^{*}(\cdot)}{\partial \omega_{h}} \equiv \frac{\Phi_{R R}^{h *}(\cdot)}{\Phi_{R R}^{j *}(\cdot)}\left(1+\frac{\partial s_{h}^{*}(\cdot)}{\partial \omega_{h}}\right) \text { for all } h, j \in\{1, \ldots, k\}
$$

and

$$
\sum_{j=1}^{k} n_{j} \frac{\partial s_{j}^{*}(\cdot)}{\partial \omega_{h}} \equiv 0
$$

Substituting (8) into (9) and rearranging yields:

$$
\frac{\partial s_{h}^{*}(\cdot)}{\partial \omega_{h}} \equiv-\frac{\sum_{i \neq h} n_{i} / \Phi_{R R}^{i *}(\cdot)}{\sum_{i=1}^{k} n_{i} / \Phi_{R R}^{i *}(\cdot)}<0
$$

Hence, thanks to the strict concavity of $\Phi$ with respect to its second argument, an increase in the wealth of a household living in jurisdiction $h$ always reduces the optimal subsidy received by this household.

If one substitutes (10) back into (8) and rearranges the expression, one gets:

$$
\frac{\partial s_{j}^{*}(\cdot)}{\partial \omega_{h}} \equiv \frac{n_{h}}{\Phi_{R R}^{j *}(\cdot)\left(\sum_{i=1}^{k} n_{i} / \Phi_{R R}^{i *}(\cdot)\right)}>0
$$

Here again, not surprisingly, the subsidy received by a household living in jurisdiction $j$ is an increasing function of the wealth of any household living in another jurisdiction.

More interesting, and relevant for Theorem 1 below, is the analogous comparative statics results that concern the relationship between a jurisdiction's optimal per capita net subsidy and its population size. One obtains in effect:

$$
\frac{\partial s_{h}^{*}(\cdot)}{\partial n_{h}} \equiv \frac{p \Phi_{p_{z} R}^{h *}(\cdot) / n_{h}^{2}\left[\sum_{i \neq h} n_{i} / \Phi_{R R}^{i *}(\cdot)\right]-s_{h}^{*}(\cdot)}{\Phi_{R R}^{h *}(\cdot)\left[\sum_{i=1}^{k} n_{i} / \Phi_{R R}^{i *}(\cdot)\right]}
$$

and

$$
\frac{\partial s_{j}^{*}(\cdot)}{\partial n_{h}}=-\frac{p \Phi_{p_{z} R}^{h *}(\cdot) / \Phi_{R R}^{h *}(\cdot)+s_{h}^{*}(\cdot)}{\Phi_{R R}^{j *}(\cdot)\left[\sum_{i=1}^{k} n_{i} / \Phi_{R R}^{i *}(\cdot)\right]}
$$


The sign of each of these two expressions, given by the sign of the numerator, cannot be determined in general. This sign depends crucially upon that of $\Phi_{p_{z} R}^{h *}$ which measures how the social marginal utility of wealth varies with the price of the public good. To understand how optimal subsidy received by jurisdiction $h$ depends upon its population size, consider the situation where the constitution maker finds optimal to give to $h$ a zero subsidy. Then, an exogenous increase in the number of households living in $h$ will reduce the optimal subsidy received by $h$ if and only if $\Phi_{p_{z} R}^{h}$ is positive. As it turns out, this simple fact is the basic ingredient of the proof of Theorem 1 below, which establishes that $\Phi_{p_{z} R}=0$ is a necessary and sufficient condition that $\Phi$ must satisfy in order for progressive net subsidies to be the solution of (7) for all distributions of wealth and population across jurisdictions.

Theorem 1. Let $U$ be a utility function in $\mathcal{U}$. Then the solution $\left\{s_{j}^{*}(\cdot)\right\}_{j=1}^{k}$ of $(7)$ is progressive for all distributions of wealth and population across jurisdictions if and only if $\Phi_{p_{z} R}=0$.

The intuition for this result goes as follows. The condition is sufficient because any redistribution of income across households of different jurisdictions does not affect the marginal (dis)utility for the public good price. If progressivity is not satisfied for two jurisdictions (that is, if a richer jurisdiction receives a larger per capita subsidy than a poorer one), it is possible to redistribute from the richer to the poorer jurisdiction without affecting the allocation of the public good. By concavity of social preferences, this redistribution is desirable.

The condition is also necessary. Suppose it was not satisfied for some $\left(p_{z}, R\right)$. Consider the federation where all jurisdictions have the same wealth and population at $R$ and $n=p / p_{z}$ respectively (for some suitable choice of $p$ ). At this allocation, all subsidies are zero. It is always possible to find a direction of change for a new federation, direction which depends on the sign of $\Phi_{p_{z} R}$, such that subsidies would not be progressive.

As mentioned above, the result of Theorem 1 which characterizes the condition under which a welfarist constitution maker always favors progressivity in the absolute sense can be rephrased in terms of the more conventional notion of relative progressivity. The key 
ingredient in this rephrasing is the definition of the function, for $j=1, \ldots, k$ :

$$
\delta_{j}^{*}: \mathbb{R}_{++} \times\left(\mathbb{N}_{++} \times \mathbb{R}_{++}\right) \rightarrow[-1, \infty
$$

by

$$
\delta_{j}^{*}\left(p, n_{1}, \omega_{1}, \ldots, n_{k}, \omega_{k}\right)=\frac{s_{j}^{*}\left(p, n_{1}, \omega_{1}, \ldots, n_{k}, \omega_{k}\right)}{\omega_{j}}
$$

for any $\left(p, n_{1}, \omega_{1}, \ldots, n_{k}, \omega_{k}\right) \in \mathbb{R}_{++} \times\left(\mathbb{N}_{++} \times \mathbb{R}_{++}\right)^{k}$. For each jurisdiction $j$, the absolute value of $\delta_{j}^{*}$ gives the fraction of jurisdiction $j$ 's per capita wealth which is received in subsidy (if $\delta_{j}^{*}$ is positive) or paid in tax (if $\delta_{j}^{*}$ is negative) that is deemed appropriate by the social planner. It is clear that $\left(\delta_{1}^{*}(\cdot), \ldots, \delta_{k}^{*}(\cdot)\right)$ is the unique solution of

$$
\begin{array}{rl}
\max _{\delta_{1}, \ldots, \delta_{k}} & W\left(V \left(p / n_{1}, 1, \omega_{1}\left(1+\delta_{1}\right), \ldots, V\left(p / n_{k}, 1, \omega_{k}\left(1+\delta_{k}\right)\right)\right.\right. \\
\text { s.t. } & \sum_{j=1}^{k} n_{j} \omega_{j} \delta_{j} \leq 0, \text { and } \delta_{j} \geq-1 \text { for all } j .
\end{array}
$$

The progressivity in the relative sense of the equalization payments system is the requirement that $\delta_{j}^{*}(\cdot) \leq \delta_{j+1}^{*}(\cdot)$ for $j=1, \ldots, k-1$. We leave to the reader the task of verifying the following fact.

Fact 1. Let $U$ be a utility function in $\mathcal{U}$. Then the solution $\left(s_{1}^{*}(\cdot), \ldots, s_{k}^{*}(\cdot)\right)$ of $(7)$ satisfies progressivity in the absolute sense if and only if the solution $\left(\delta_{1}^{*}(\cdot), \ldots, \delta_{k}^{*}(\cdot)\right)$ of $(14)$ satisfies progressivity in the relative sense.

\section{Interpretation for a mean-of-order $\mathrm{r}$ constitution maker}

We provide in this section some implications for the households' utility function, of the requirement of additive separability of $\Phi$ characterized in Theorem 1 . The next theorem provides these restrictions for the case where the constitution maker aggregates households' utilities by a mean-of-order $r$ social welfare function defined above. 
Theorem 2. Let $U$ be utility function in $\mathcal{U}$ and let the social welfare function $W$ be meanof-order $r$ as defined above where $r \in-\infty, 1]$. Then, $\Phi_{p_{z} R}\left(p_{z}, R\right)=0$ for every $\left(p_{z}, R\right) \in \mathbb{R}_{+}^{2}$ if and only if each household's indirect utility function can be written as

$$
\left.V\left(p_{z}, p_{c}, R\right)=\left[\Upsilon_{1}^{r}\left(p_{z}, p_{c}\right)+\Upsilon_{2}^{r}\left(p_{c}, R\right)\right]^{\frac{1}{r}} \text { for } r \in-\infty, 1\right] \backslash\{0\}
$$

and

$$
V\left(p_{z}, p_{c}, R\right)=\left[\Upsilon_{1}^{0}\left(p_{z}, p_{c}\right) \Upsilon_{2}^{0}\left(p_{c}, R\right)\right] \text { for } r=0
$$

This theorem illustrates the strength of the condition of additive separability of the social objective in terms of its implication on households' preferences for the public and the private goods. As Theorem 2 makes clear, these indirect preferences must be additively separable with respect to the price of the public good and the household wealth. Furthermore, Theorem 2 indicates that the numerical representation of these indirect preferences that the constitution maker must use in order to achieve its objective must be the additive representation of these preferences raised at the power $1 / r$. That this additive separability of the household's indirect preference is a significant restriction is now further emphasized by considering that the household's direct utility function used to define the additively separable indirect utility function is itself additively separable.

Theorem 3. Let $U$ be a direct utility function in $\mathcal{U}_{A}$. Then,

$$
V\left(p_{z}, p_{c}, R\right)=\Upsilon^{0}\left(p_{z}, p_{c}\right)+\Upsilon^{1}\left(p_{c}, R\right)
$$

for some functions $\Upsilon^{0}: \mathbb{R}_{++}^{2} \rightarrow \mathbb{R}$ and $\Upsilon^{1}: \mathbb{R}_{++}^{2} \rightarrow \mathbb{R}$ continuous and homogeneous of degree 0 if and only if $U$ can be written as

$$
U(z, c)=b \ln z+h(c)
$$

for all $(z, c) \in \mathbb{R}_{+}^{2}$ and some strictly positive real number $b$.

Hence, within the class of additively separable utility functions, only those that are logarithmic with respect to public good consumption can give rise to indirect utility functions 
for which the marginal utility of wealth is independent from the (Lindahl) price of public good. In conjunction with Theorems 1 and 2, this implies that if a welfarist social planner uses primal utility functions that are additively separable, it is only when these utilities are logarithmic in the public good that the planner always considers optimal to set up a progressive equalization payments scheme.

In the proof of Theorem 3, some use has been made of the property of strict concavity of the utility function (in particular the property that $\partial^{2} h(c) / \partial^{2} c<0$ ). This property rules out the possibility for the utility function to be quasi-linear (linear in the private good, concave in the public good), a property commonly assumed in public good provision problems. If one is willing to weaken the requirement of strict concavity of the utility function to that of concavity (which allows for quasi-linearity), then one can obtain the following minor amendment to Theorem 3.

Theorem 4. Let $U$ be an additively separable, derivable, monotonically increasing, and concave direct utility function. Then,

$$
V\left(p_{z}, p_{c}, R\right)=\Upsilon^{0}\left(p_{z}, p_{c}\right)+\Upsilon^{1}\left(p_{c}, R\right)
$$

for some functions $\Upsilon^{0}: \mathbb{R}_{++}^{2} \rightarrow \mathbb{R}$ and $\Upsilon^{1}: \mathbb{R}_{++}^{2} \rightarrow \mathbb{R}$ continuous and homogeneous of degree 0 if and only if either $U$ can be written as

$$
U(z, x)=b \ln z+h(c)
$$

for all $(z, c) \in \mathbb{R}_{+}^{2}$ and some strictly positive real number $b$, or $U$ is linear with respect to the private good, that is, $U$ can be written as

$$
U(z, x)=f(z)+a c
$$

for all $(z, c) \in \mathbb{R}_{+}^{2}$ and some strictly positive real number a.

\section{Conclusion}

This paper provides a necessary and sufficient condition that an individual indirect utility 
function must satisfy in order for the choice of an equalization payments system made by a welfarist constitution maker who uses an additively separable social welfare function to be progressive. The condition requires the indirect utility function to be additively separable with respect to the (Lindahl) price of public good and wealth. The condition is rather strong since it implies, for instance, that if the direct utility function is additively separable, it must be either logarithmic with respect to the public good or linear with respect to the private good.

These results are derived in a world where citizens are not allowed to move from one jurisdiction to another. A natural extension of the research, pursued in the companion paper Gravel and Poitevin (2004), would be the analysis of the structure of per capita subsidies that would be chosen by a welfarist constitution maker who would allow households to change jurisdiction in order to get their most preferred allocation of public good and taxes.

From the perspective of Lemma 1, it is not surprising to find that progressivity is not a genuine property that an ideal welfarist constitution maker would like to impose on an equalization payments scheme in a federation. After all, the notion of progressivity of a tax system (or an equalization payments scheme) considered in this paper, and for that matter in most of the literature we are aware of, is justified by the equalizing impact that this system is supposed to have on the distribution of income. A progressive tax system (or equalization payments scheme) is simply a system which guarantees that the after-tax income distribution is unambiguously more equal than the before-tax one. Yet the standard definition of what it means for an income distribution to be unambiguously more equal than another is based on the Lorenz domination criterion (in its absolute or relative version). While there are sound and well-known arguments (see, for instance, Atkinson (1970) or Sen (1973)) in favor of using Lorenz domination criteria in a world where households are identical in every respect other than income, these arguments collapse in the world considered herein where households living in different jurisdictions differ both in private and public consumption. Why after all should we favor transferring wealth from a province with a high per capita GDP and with a small population to a poorer but more populous one? Since the latter has a cost 
advantage in producing the public good, it is not clear that it should receive subsidy from the former. What is needed in order to appraise progressivity in the current context is a theory of multidimensional inequality measurement, one which would deal with inequality that arises both from the distribution of the private good and the public good. And it is fair to say that, despite some interesting attempts, the most relevant to the current context being Atkinson and Bourguignon (1982), multidimensional inequality measurement has not reached the state of development of its unidimensional counterpart. Developing methods for normatively appraising the multidimensional inequalities that arise both from public good and private good consumption should clearly be a high priority for future research.

If the mere conclusion that an optimal equalization payments scheme need not be progressive in the usual (unidimensional) sense of the word is not, in itself surprising, it remains that many actual federal systems are designed with this standard notion of progressivity. The main contribution of this paper should thus be seen as the identification of the exact set of conditions that citizens' preferences must satisfy in order for progressivity to be rationalizable from a welfarist perspective.

\section{References}

Aczél, M. and A. Pfingsten, "Constituent Sensitive Public Fund Sharing," in W.E. Diewert, K Spreman, and K. Sterling, eds., Mathematical Modelling in Economics, Springer Verlag, 1993, pp. 2-10.

Atkinson, A. B. and F. Bourguignon, "The Comparison of Multi-Dimensioned Distribution of Economic Status," Review of Economic Studies, 1982, 49, 183-201.

Atkinson, A.B., "On the Measurement of Inequality," Journal of Economic Theory, 1970, 2, 244-263.

Blackorby, C. and D. Donaldson, "Ratio-Scale and Translation-Scale Full Interpersonnal Comparability Without Domain Restrictions: Admissible Social Evaluation Func- 
tions," International Economic Review, 1982, 23, 249-268.

, W. Bossert, and D. Donaldson, "Utilitarianism and the Theory of Justice," in K. Arrow, A. Sen, and K. Suzumura, eds., Handbook of Social Choice and Welfare, Elsevier, 2001.

Buhl, H. U. and A. Pfingsten, "On the Distribution of Public Funds," European Journal of Political Economy, 1990, 6, 363-376.

Eichhorn, W., H. Funke, and W. F. Richter, "Tax Progression and Inequality of Income Distribution," Journal of Mathematical Economics, 1984, 13, 127-131.

Gravel, N. and M. Poitevin, "What Is the Best Jurisdiction Structure?," 2004. Mimeo, IDEP.

Jakobsson, U., "On the Measurement of the Degree of Progression," Journal of Public Economics, 1976, 8, 161-168.

Kolm, S. C., "Unequal Inequalities I," Journal of Economic Theory, 1976, 12, 416-442.

Moyes, P., "A New Concept of Lorenz Domination," Economics Letter, 1987, 23, 203-207. , "A Note on Minimally Progressive Taxation and Absolute Income Inequality," Social Choice and Welfare, 1988, 5, 227-234.

_ _ , "Inequality Reducing and Inequality Preserving Transformations of Income: Symmetric and Individualistic Transformations," Journal of Economic Theory, 1994, 63, 271-298.

Sen, A. K., On Economic Inequality, Oxford, Clarendon, 1973.

Thon, D., "Redistributive Properties of Progressive Taxation," Mathematical Social Science, 1987, 14, 185-191. 


\section{APPENDIX}

\section{Proof of Lemma 1}

Assume by contradiction that

$$
\left\{z_{j}^{M}\left(p / n_{j}, 1, \omega_{j}+s_{j}^{*}(\cdot)\right), \omega_{j}-c_{j}^{M}\left(p / n_{j}, 1, \omega_{i}+s_{j}^{*}(\cdot)\right)\right\}_{j=1}^{k}
$$

does not solve (3), that is, assume that there exists $\left(\widehat{z}_{1}, \widehat{T}_{1}, \ldots, \widehat{z}_{k}, \widehat{T}_{k}\right)$ satisfying $\sum_{j=1}^{k} p \widehat{z}_{j} \leq \sum_{j=1}^{k} n_{j} \widehat{T}_{j}$, $\widehat{T}_{j} \leq \omega_{j}$ and $\widehat{z}_{j} \geq 0$ for all $j$ such that

$$
W\left(U\left(\widehat{z}_{1}, \omega_{1}-\widehat{T}_{1}\right), \ldots, U\left(\widehat{z}_{k}, \omega_{k}-\widehat{T}_{k}\right)\right)>W\left(V\left(p / n_{1}, 1, \omega_{1}+s_{1}^{*}(\cdot)\right), \ldots, V\left(p / n_{k}, 1, \omega_{k}+s_{k}^{*}(\cdot)\right)\right) .
$$

Without loss of generality, since program (3) has a solution in which constraint (2) binds, one can assume that $\sum_{j=1}^{k} p \widehat{z}_{j}=\sum_{j=1}^{k} n_{j} \widehat{T}_{j}$. Define, for every $j, \widehat{s}_{j}=p \widehat{z}_{j} / n_{j}-\widehat{T}_{j}$ and $\widehat{c}_{j}=\omega_{j}-\widehat{T}_{j}$. Clearly, $p \widehat{z}_{j} / n_{j}+\widehat{c}_{j} \leq \omega_{j}+\widehat{s}_{j}$. By definition of the indirect utility function, one has, for every $j$

$$
V\left(p / n_{j}, 1, \omega_{j}+\widehat{s}_{j}\right) \geq U\left(\widehat{z}_{j}, \omega_{j}-\widehat{T}_{j}\right)
$$

and, since $W$ is monotonically increasing,

$$
W\left(V\left(p / n_{1}, 1, \omega_{1}+\widehat{s}_{1}\right), \ldots, V\left(p / n_{k}, 1, \omega_{k}+\widehat{s}_{k}\right)\right) \geq W\left(U\left(\widehat{z}_{1}, \omega_{1}-\widehat{T}_{1}\right), \ldots, U\left(\widehat{z}_{k}, \omega_{k}-\widehat{T}_{k}\right)\right)
$$

Since $\widehat{s}_{j} \geq-\omega_{j}$ for all $j$ and $\sum_{j=1}^{k} \widehat{s}_{j}=0$, this inequality, together with (15), contradicts the definition of $\left\{s_{j}^{*}\right\}_{j=1}^{k}$ as the solution to program (4).

\section{Proof of Theorem 1}

- Sufficiency. Assume $\Phi_{p_{z} R}=0$. Then, one can write $\Phi\left(p_{z}, R\right)=\Upsilon\left(p_{z}\right)+\Psi(R)$ for some functions $\Upsilon: \mathbb{N}_{++} \rightarrow \mathbb{R}$ monotonically decreasing and quasi-convex, and $\Psi: \mathbb{R}_{+} \rightarrow \mathbb{R}$ monotonically increasing and strictly concave (since $V$ is concave with respect to wealth and $g$ is concave). By contradiction, consider any $\left(p, n_{1}, \omega_{1}, \ldots, n_{k}, \omega_{k}\right) \in \mathbb{R}_{++} \times\left(\mathbb{N}_{++} \times \mathbb{R}_{++}\right)^{k}$ and assume that the solution $\left(s_{1}^{*}(\cdot), \ldots, s_{k}^{*}(\cdot)\right)$ of $(7)$ involves $s_{j}^{*}\left(p, n_{1}, \ldots, n_{k}, \omega_{1}, \ldots, \omega_{k}\right)>s_{j+1}^{*}\left(n_{1}, \ldots, n_{k}, \omega_{1}, \ldots, \omega_{k}\right)$ for some $j \in\{1, \ldots, k-1\}$. Consider reducing the net per capita subsidy of jurisdiction $j$ by $\delta \equiv\left(\omega_{j}-\omega_{j+1}+s_{j}^{*}(\cdot)-s_{j+1}^{*}(\cdot)\right) / 2>0$ and increasing that of jurisdiction $j+1$ by the same amount. This change in per capita net subsidies obviously respects the constraints of program (7). Notice in particular that

$$
\omega_{j}+s_{j}^{*}(\cdot)>\omega_{j}+s_{j}^{*}(\cdot)-\delta=\omega_{j+1}+s_{j+1}^{*}(\cdot)+\delta>\omega_{j+1}+s_{j+1}^{*}(\cdot)
$$


Now:

$$
\begin{aligned}
& \sum_{h \neq j, j+1} n_{h} \Phi\left(p / n_{h}, \omega_{h}+s_{h}^{*}(\cdot)\right)+n_{j} \Phi\left(p / n_{j}, \omega_{j}+s_{j}^{*}(\cdot)-\delta\right)+ \\
& n_{j+1} \Phi\left(p / n_{j+1}, \omega_{j}+s_{j}^{*}(\cdot)-\delta\right)-\sum_{h=1}^{k} n_{h} \Phi\left(p / n_{h}, \omega_{h}+s_{h}^{*}(\cdot)\right) \\
= & n_{j} \Phi\left(p / n_{j}, \omega_{j}+s_{j}^{*}(\cdot)-\delta\right)+n_{j+1} \Phi\left(p / n_{j+1}, \omega_{j}+s_{j}^{*}(\cdot)-\delta\right) \\
& -n_{j} \Phi\left(p / n_{j}, \omega_{j}+s_{j}^{*}(\cdot)\right)-n_{j+1} \Phi\left(p / n_{j+1}, \omega_{j+1}+s_{j+1}^{*}(\cdot)\right) \\
= & n_{j} \Psi\left(\omega_{j}+s_{j}^{*}(\cdot)-\delta\right)+n_{j+1} \Psi\left(\omega_{j}+s_{j}^{*}(\cdot)-\delta\right)-n_{j} \Psi\left(\omega_{j}+s_{j}^{*}(\cdot)\right)-n_{j+1} \Psi\left(\omega_{j+1}+s_{j+1}^{*}(\cdot)\right) \\
> & 0
\end{aligned}
$$

by the concavity of $\Psi$. This gives us the required contradiction that $\left(s_{1}^{*}(\cdot), \ldots, s_{k}^{*}(\cdot)\right)$ is a solution of program (7).

- Necessity. Assume that $\Phi_{p_{z} R}\left(p_{z}, R\right) \neq 0$ for some $\left(p_{z}, R\right) \in \mathbb{R}_{++}^{2}$. Consider the federation where, for some strictly positive real number $p$ and strictly positive integer $\widetilde{n}$ satisfying $p_{z}=p / \widetilde{n}$, one has $\left(p, n_{1}, \omega_{1}, \ldots, n_{k}, \omega_{k}\right)=(p, \widetilde{n}, R, \widetilde{n}, R, \ldots, \widetilde{n}, R)$. The optimal subsidies that solve (7) for this

federation are $s_{j}^{*}(p, \widetilde{n}, R, \widetilde{n}, R, \ldots, \tilde{n}, R)=0$ for all $j$. Assume first that $\Phi_{p_{z} R}(1 / \widetilde{n}, R)>0$, and consider increasing by a suitably small and strictly positive $\varepsilon$ the number of inhabitants in some jurisdiction $h$. Using (13), we know that the subsidies received in jurisdictions $j \neq h$ increase and become positive while the subsidies received in $h$ become negative. Using (12) and (13), we have that

$$
s_{h}^{*}(p, \widetilde{n}, R, \ldots, \widetilde{n}, R, \widetilde{n}+\varepsilon, R, \widetilde{n}, R, \ldots, \widetilde{n}, R)<0<s_{j}^{*}\left(\widetilde{n}, R, \ldots, \widetilde{n}, R, \widetilde{n}+\varepsilon^{*}, R, \widetilde{n}, R, \ldots, \widetilde{n}, R\right)
$$

for all $j \neq h$. A small enough increase of individual $j$ 's wealth would give us, in view of (10) and the continuity of the optimal response functions $s_{j}^{*}$, the required violation of progressivity. The argument for the case where $\Phi_{p_{z} R}(1 / \widetilde{n}, R)<0$ is similar.

\section{Proof of Theorem 2}

Assume first that $r \in-\infty, 1] \backslash\{0\}$. Using the definition of the mean-of-order $r$ function provided by (6), we have

$$
\Phi_{p_{z} R}\left(p_{z}, R\right)=0 \quad \Leftrightarrow
$$




$$
(r-1) V\left(p_{z}, 1, R\right)^{r-2} \frac{\partial V\left(p_{z}, 1, R\right)}{\partial p_{z}} \frac{\partial V\left(p_{z}, 1, R\right)}{\partial R}+V\left(p_{z}, 1, R\right)^{r-1} \frac{\partial^{2} V\left(p_{z}, 1, R\right)}{\partial p_{z} \partial R}=0 .
$$

For $r=1$ (utilitarianism), this equality amounts to $\partial^{2} V\left(p_{z}, 1, R\right) / \partial p_{z} \partial R=0$ which is equivalent to the additive separability of the indirect utility function with respect to $p_{z}$ and $R$. If $r<1$ (but $r \neq 0)$, this equality can be written as

$$
(1-r) \frac{\partial V\left(p_{z}, 1, R\right) / \partial p_{z}}{V\left(p_{z}, 1, R\right)}=\frac{\partial^{2} V\left(p_{z}, 1, R\right) / \partial p_{z} \partial R}{\partial V\left(p_{z}, 1, R\right) / \partial R}
$$

or

$$
\frac{\partial\left(\ln V\left(p_{z}, 1, R\right)^{1-r}\right)}{\partial p_{z}}=\frac{\partial\left(\ln \partial V\left(p_{z}, 1, R\right) / \partial R\right)}{\partial p_{z}} .
$$

This is a first-order partial differential equation assumed to hold for every $\left(p_{z}, R\right) \in \mathbb{R}_{+}^{2}$. Define $\widehat{V}: \mathbb{R}_{+}^{2} \rightarrow \mathbb{R}_{+}$by $\widehat{V}\left(p_{z}, R\right)=V\left(p_{z}, 1, R\right)$. It can be checked easily that a solution to the partial differential equation (16) is given by

$$
\widehat{V}\left(p_{z}, R\right)=\left(\widehat{\Upsilon}_{1}^{r}\left(p_{z}\right)+\widehat{\Upsilon}_{2}^{r}(R)\right)^{\frac{1}{r}}
$$

for some functions $\widehat{\Upsilon}_{1}^{r}: \mathbb{R}_{+} \rightarrow \mathbb{R}_{+}$and $\widehat{\Upsilon}_{2}^{r}: \mathbb{R}_{+} \rightarrow \mathbb{R}_{+}$(both depending upon $r$ ). By usual regularity arguments for partial differential equations, this solution is unique (up to irrelevant constant terms).

For the case where $r=0$, using (6), we have that

$$
\begin{aligned}
\Phi_{p_{z} R}\left(p_{z}, R\right)=0 & \Leftrightarrow \frac{\partial^{2}\left(\ln \widehat{V}\left(p_{z}, R\right)\right)}{\partial p_{z} \partial R}=0 \\
& \Leftrightarrow \frac{\partial \widehat{V}\left(p_{z}, R\right) / \partial p_{z}}{\widehat{V}\left(p_{z}, R\right)}=\frac{\partial^{2} \widehat{V}\left(p_{z}, R\right) / \partial p_{z} \partial R}{\partial \widehat{V}\left(p_{z}, R\right) / \partial R} \\
& \Leftrightarrow \frac{\partial\left(\ln \widehat{V}\left(p_{z}, R\right)\right)}{\partial p_{z}}=\frac{\partial\left(\ln \partial \widehat{V}\left(p_{z}, R\right) / \partial R\right)}{\partial p_{z}},
\end{aligned}
$$

a partial differential equation, a (unique by standard arguments) solution of which is

$$
\widehat{V}\left(p_{z}, R\right)=\Upsilon_{1}^{0}\left(p_{z}\right) \Upsilon_{2}^{0}(R)
$$

for some functions $\widehat{\Upsilon}_{1}^{0}: \mathbb{R}_{+} \rightarrow \mathbb{R}_{+}$and $\widehat{\Upsilon}_{2}^{0}: \mathbb{R}_{+} \rightarrow \mathbb{R}_{+}$. We note finally that, since $V$ is homogeneous of degree 0 , we have $\widehat{V}\left(p_{z}, R\right)=V\left(\widehat{p}_{z}, \widehat{p}_{c}, \widehat{R}\right)$ for every $\left(\widehat{p}_{z}, \widehat{p}_{c}, \widehat{R}\right) \in \mathbb{R}_{++}^{3}$ such that $p_{z}=\widehat{p}_{z} / \widehat{p}_{c}$ and $R=\widehat{R} / \widehat{p}_{c}$. Hence, the indirect utility function must have the form (for every $\left(p_{z}, p_{c}, R\right) \in \mathbb{R}_{++}^{3}$ )

$$
\left.V\left(p_{z}, p_{c}, R\right)=\left[\Upsilon_{1}^{r}\left(p_{z}, p_{c}\right)+\Upsilon_{2}^{r}\left(p_{c}, R\right)\right]^{\frac{1}{r}} \text { for } r \in-\infty, 1\right] \backslash\{0\}
$$


and

$$
V\left(p_{z}, p_{c}, R\right)=\Upsilon_{1}^{0}\left(p_{z}, p_{c}\right) \Upsilon_{2}^{0}\left(p_{c}, R\right) \text { for } r=0
$$

where, for all $r \in-\infty, 1], \Upsilon_{1}^{r}: \mathbb{R}_{++}^{2} \rightarrow \mathbb{R}_{+}$is a twice continuously differentiable function that is homogeneous of degree 0 and monotonically decreasing (resp. increasing) with respect to each of its two arguments if $r \geq 0$ (resp. $<0$ ), and $\Upsilon_{2}^{r}: \mathbb{R}_{++}^{2} \rightarrow \mathbb{R}_{+}$is a twice continuously differentiable function that is homogeneous of degree 0, monotonically decreasing (resp. increasing) in its first argument and monotonically increasing (resp. decreasing) in its second argument if $r \geq 0$ (resp. < $0)$.

\section{Proof of Theorem 3}

The sufficiency part being straightforward, we only provide the proof of necessity. We first notice that, under additive separability of $U$,

$$
\frac{\partial^{2} V\left(\bar{p}_{z}, \bar{p}_{c}, \bar{R}\right)}{\partial p_{z} \partial R}=0 \text { for every }\left(\bar{p}_{z}, \bar{p}_{c}, \bar{R}\right) \in \mathbb{R}_{++}^{3} \Leftrightarrow \frac{\partial c^{M}\left(\bar{p}_{z}, \bar{p}_{c}, \bar{R}\right)}{\partial p_{z}}=0 .
$$

To see this, note that, by definition of the indirect utility function, and under additive separability of $U$ :

$$
\begin{aligned}
\frac{\partial V\left(\bar{p}_{z}, \bar{p}_{c}, \bar{R}\right)}{\partial R}= & \frac{\partial f\left(z^{M}\left(\bar{p}_{z}, \bar{p}_{c}, \bar{R}\right)\right)}{\partial z} \frac{\partial z^{M}\left(\bar{p}_{z}, \bar{p}_{c}, \bar{R}\right)}{\partial R} \\
& +\frac{\partial h\left(\bar{R} / \bar{p}_{c}-\bar{p}_{z} / \bar{p}_{c} z^{M}\left(\bar{p}_{z}, \bar{p}_{c}, \bar{R}\right)\right)}{\partial c}\left(\frac{1-\bar{p}_{z} \partial z^{M}\left(\bar{p}_{z}, \bar{p}_{c}, \bar{R}\right) / \partial R}{\bar{p}_{c}}\right) \\
= & \frac{\partial h\left(c^{M}\left(\bar{p}_{z}, \bar{p}_{c}, \bar{R}\right)\right)}{\partial c}\left(\frac{1}{\bar{p}_{c}}\right)
\end{aligned}
$$

for every $\left(\bar{p}_{z}, \bar{p}_{c}, \bar{R}\right) \in \mathbb{R}_{++}^{3}$, thanks to the first-order conditions of the consumer's problem satisfied by Marshallian demands. Hence, as $\bar{p}_{c}$ is strictly positive, the condition $\partial^{2} V\left(\bar{p}_{z}, \bar{p}_{c}, \bar{R}\right) / \partial p_{z} \partial R=0$ is equivalent to the condition

$$
\frac{\partial^{2} h\left(c^{M}\left(\bar{p}_{z}, \bar{p}_{c}, \bar{R}\right)\right)}{\partial^{2} c} \frac{\partial c^{M}\left(\bar{p}_{z}, \bar{p}_{c}, \bar{R}\right)}{\partial p_{z}}=0
$$

Since $\partial^{2} h\left(c^{M}\left(\bar{p}_{z}, \bar{p}_{c}, \bar{R}\right)\right) / \partial^{2} c<0$, thanks to the strict concavity assumption, this is in turn equivalent to the condition that $\partial c^{M}\left(\bar{p}_{z}, \bar{p}_{c}, \bar{R}\right) / \partial p_{z}=0$ everywhere. Now the Marshallian demand function $c^{M}$ is locally characterized by the first-order condition of the standard consumer problem:

$$
-\frac{\partial f\left(R / p_{z}-\left(p_{c} / p_{z}\right) c^{M}(\cdot)\right)}{\partial z} \frac{p_{c}}{p_{z}}+\frac{\partial h\left(c^{M}(\cdot)\right)}{\partial c} \equiv 0 .
$$


Differentiating this (local) identity with respect to $p_{z}$ and rearranging terms yield

$$
\frac{\partial c^{M}\left(p_{z}, p_{c}, R .\right)}{\partial p_{z}} \equiv \frac{-\left(p_{c} / p_{z}^{2}\right)\left(z^{M}(\cdot) \partial^{2} f(\cdot) / \partial^{2} z+\partial f(\cdot) / \partial z\right)}{\left(p_{c} / p_{z}\right)^{2} \partial^{2} f(\cdot) / \partial^{2} z+\partial^{2} h(\cdot) / \partial^{2} c}
$$

Requiring $\partial c^{M}\left(p_{z}, p_{c}, R.\right) / \partial p_{z}=0$ for every $\left(p_{z}, p_{c}, R\right) \in \mathbb{R}_{++}^{3}$ amounts (since the denominator of (17) is strictly negative because of the strict concavity of $U$ ) to requiring

$$
z \frac{\partial^{2} f(z)}{\partial^{2} z}+\frac{\partial f(z)}{\partial z}=0
$$

to hold for every $z \in \mathbb{R}_{++}$. The partial differential equation (18) can also be written as

$$
\frac{\partial^{2} f(z) / \partial^{2} z}{\partial f(z) / \partial z}=-\frac{1}{z}
$$

or

$$
\frac{\partial(\ln \partial f(z) / \partial z)}{\partial z}=-\frac{1}{z} \Leftrightarrow \alpha+\ln \frac{\partial f(z)}{\partial z}=-\ln z+\beta
$$

for some real numbers $\alpha$ and $\beta$. Taking the exponential on both sides and rearranging terms yield

$$
\frac{\partial f(z)}{\partial z}=\frac{e^{\beta}}{e^{\alpha} z}
$$

or

$$
f(z)=\frac{e^{\beta}}{e^{\alpha}} \ln z
$$

